\title{
Neutralizing activities of human immunoglobulin derived from donors in Japan against mosquito- borne flaviviruses, Japanese encephalitis virus, West Nile virus, and dengue virus
}

\author{
This article was published in the following Dove Press journal: \\ Biologics: Targets and Therapy \\ 7 July 2016 \\ Number of times this article has been viewed
}

\author{
Mikihiro Yunoki ${ }^{1-3}$ \\ Takeshi Kurosu² \\ Ritsuko Kubota Koketsu ${ }^{2,4}$ \\ Kazuo Takahashi ${ }^{5}$ \\ Yoshinobu Okuno ${ }^{4}$ \\ Kazuyoshi Ikuta ${ }^{2,4}$ \\ 'Research and Development Division, \\ Japan Blood Products Organization, \\ Tokyo, ${ }^{2}$ Department of Virology, \\ Research Institute for Microbial \\ Diseases, Osaka University, Osaka, \\ ${ }^{3}$ Pathogenic Risk Evaluation, Graduate \\ School of Veterinary Medicine, \\ Rakuno Gakuen University, Hokkaido, \\ ${ }^{4}$ Research and Development \\ Division, The Research Foundation \\ for Microbial Diseases of Osaka \\ University, Kagawa, ${ }^{5}$ Osaka Prefectural \\ Institute of Public Health, Osaka, \\ Japan
}

Correspondence: Mikihiro Yunok Research and Development Division, Japan Blood Products Organization, 7F World Trade Center Building, 2-4-I Hamamatsu-cho, Minato-ku, Tokyo 105-6I07, Japan

Email yunoki-mikihiro@jbpo.or.jp

Ritsuko Kubota Koketsu Department of Virology, Research Institute for Microbial Diseases, Osaka University, 3-I Yamadaoka, Suita, Osaka 565-087I, Japan

Email rkoketsu@mail.biken.or.jp

\begin{abstract}
Japanese encephalitis virus (JEV), West Nile virus (WNV), and dengue virus (DenV) are causal agents of Japanese encephalitis, West Nile fever, and dengue fever, respectively. JEV is considered to be indigenized and widespread in Japan, whereas WNV and DenV are not indigenized in Japan. Globulin products seem to reflect the status of the donor population according to antivirus neutralization activity. However, the anti-JEV, -WNV, and -DenV neutralization activities of globulin products derived from donors in Japan have not been clarified. Furthermore, potential candidates for the development of an effective immunotherapeutic drug for encephalitis caused by JEV, WNV, or DenV have also not been identified. Therefore, the aim of this study was to determine the overall status of the donor population in Japan based on globulin products by evaluating anti-JEV, -WNV, and -DenV neutralizing activities of intravenous immunoglobulin. Overall, intravenous immunoglobulin products showed stable neutralizing activity against JEV but showed no or only weak activity against WNV or DenV. These results suggest that the epidemiological level against WNV and DenV in the donor population of Japan is still low, suggesting that these viruses are not yet indigenized. In addition, JEV vaccinations and/or infections in the donor population do not induce a cross-reactive antibody against WNV. Keywords: Japanese encephalitis virus, West Nile virus, dengue virus, intravenous immunoglobulin, neutralization
\end{abstract}

\section{Introduction}

Japanese encephalitis virus (JEV), West Nile virus (WNV), and dengue virus (DenV) are causal agents of Japanese encephalitis, West Nile fever, and dengue fever, respectively. These viruses are transmitted through mosquitoes and are all members of the family Flaviviridae. ${ }^{1} \mathrm{JEV}$ has been indigenized and is widespread in Japan, and therefore a JEV vaccine program has been in operation since 1954 in Japan. ${ }^{2,3}$ Currently, Japanese children are inoculated with the vaccine several times; consequently, the donor population in Japan has anti-JEV immunoglobulin G. Nevertheless, there were 85 cases of Japanese encephalitis reported from 1999 to $2013 .{ }^{4}$ However, there was only one case of West Nile fever reported from 2002 to 2013 and 1,455 cases of dengue fever reported from 1999 to 2013 in Japan. ${ }^{4}$ These cases were all considered to have been due to the importation of the viruses by travelers. In addition, JEV has 
been detected from mosquitoes in Japan, whereas WNV and DenV have not been detected. The existence of virus-positive mosquitoes suggests that the virus circulates among host vectors. Therefore, it is generally believed that WNV and DenV are not indigenized in Japan at present. This situation suggests that the donor population in Japan has been stimulated by JEV but not by WNV or DenV, except for a few individuals harboring anti-WNV or -DenV who became infected in other countries. In 2014, indigenized dengue cases were reported in the Tokyo area, although the last reported indigenized cases were in the $1940 \mathrm{~s} ;{ }^{5}$ these cases all resolved within a few months. In addition, the outbreak highlighted the risk of indigenization of DenV or the occurrence of another outbreak in the near future. However, the occurrence of subclinical infections of WNV or DenV in Japan remains unknown.

Intravenous immunoglobulin (IVIG) is manufactured from pooled plasma derived from over 10,000 donors. Therefore, analysis of IVIG can reflect the overall epidemiological status for a donor population, region, and period. Evaluations of the epidemiological situation of these viruses in Japan could suggest that the donated blood harbors significant anti-JEV activity and no or weak anti-WNV and -DenV activities. Alternatively, anti-WNV and -DenV activity may be detected if there are many latent asymptomatic cases in Japan. A comparison study was performed to evaluate the anti-WNV titer of IVIGs derived from plasma collected in the US before and after the occurrence of a WNV outbreak. In this case, the anti-WNV neutralization titers of IVIGs before and after indigenization were $21 \pm 1$ and $208 \pm 40$ (mean \pm standard error of the mean), respectively, clearly reflecting that a change in epidemiological status can be detected in IVIGs. ${ }^{6}$ However, in Japan, the current status of the neutralizing activity against JEV, WNV, and DenV of IVIG remains unknown. Accordingly, the overall indigenized situation of the donor population in Japan is still unclear. Furthermore, potential components that could serve as an effective immunotherapeutic drug for encephalitis caused by JEV, WNV, or DenV may be discovered by evaluating the IVIG neutralizing activity. Hence, the aim of the present study was to evaluate the neutralization activity against JEV, WNV, and four serotypes of DenV of IVIGs manufactured from 2000 to 2010 that were derived from donor plasma in Japan.

\section{Materials and methods}

The IVIG Venoglobulin ${ }^{\circledR}$ IH 5\% I.V. (Japan Blood Products Organization, Tokyo, Japan; formerly Benesis Corporation, Osaka, Japan) derived from donors in Japan was used in this study. This study was approved by the ethical committee of Japan Blood Products Organization (formerly Benesis
Corporation). A total of 21 lots, manufactured from 2000 to 2010, were tested with the following viruses: JEV (Beijing strain), WNV (New York strain), DenV type 1 (clinical isolate), type 2 (New Guinea $\mathrm{C}$ strain), type 3 (clinical isolate), and type 4 (clinical isolate). The neutralization assay was conducted as previously reported. ${ }^{7-9}$ In brief, IVIG samples were diluted with culture media (JEV and WNV: tenfold followed by a twofold serial dilution; DenV: fourfold serial dilution). Diluted samples were incubated with a virus (100 focus-forming units each), and then the mixture was inoculated to Vero cells as viral detector cells. After adsorption, the mixture was removed, and the cells were washed with culture media and then cultured with media for a preset number of days. Assays of WNV and DenV were conducted in culture media supplemented with (DenV) or without (JEV and WNV) methyl cellulose. Furthermore, experiments using WNV were also performed without removing the neutralizing mixture and without the washing step after adsorption for comparison of culture with globulin for detection of neutralizing antibodies, which inhibit several steps of viral replication. Infected cells were counted using focus assays. The titer was determined based on the maximum dilution of a decreasing focus rate of $>50 \%$.

\section{Results and discussion}

IVIG lots derived from donors in Japan, manufactured from 2000 to 2010, showed an anti-JEV neutralizing titer of 160 , whereas no neutralizing activity against WNV or the DenVs was observed (Table 1). In addition, WNV was also cultured with human globulin, which allows for the virus to undergo multiple cycles of replication, and thus enables detection of neutralizing antibodies in the human globulin that inhibit several steps of viral replication. In this condition, very weak neutralizing activity was observed. Thus, the experiment of virus incubation without globulin could specifically reflect neutralizing activity against virus attachment and entry to host cells, whereas incubation with globulin reflects neutralizing activity from the stage of virus attachment to the release of progeny viruses. These results indicated that the IVIG showed no or very weak neutralizing activity against WNV for all of these stages.

The IVIG lots showed significant and stable neutralizing titers against JEV throughout the $>10$-year period of collection. This result reflects the accumulation of natural infections and vaccination in the donor population. On the other hand, no neutralizing activities of anti-WNV and DenV types 1 to 4 were detected. WNV belongs to the JEV group. ${ }^{1}$ Therefore, it is expected that JEV vaccination or infection would induce a cross-reactive antibody against WNV. An 
Table I Neutralizing titers against JEV, WNV, and DenV of IVIG manufactured from 2000 to 2010 in Japan

\begin{tabular}{|c|c|c|c|c|c|c|c|}
\hline & \multicolumn{7}{|c|}{ Neutralization titer } \\
\hline & \multicolumn{3}{|l|}{ JEV group } & \multicolumn{4}{|l|}{ DenV group } \\
\hline & \multirow[t]{2}{*}{ JEV } & \multicolumn{2}{|l|}{ WNV } & \multicolumn{4}{|l|}{ DenV } \\
\hline & & & & Type I & Type 2 & Type 3 & Type 4 \\
\hline Year manufactured & $\begin{array}{l}\text { Culture } \\
\text { condition: } \\
\text { without IgG }\end{array}$ & $\begin{array}{l}\text { Culture } \\
\text { condition: } \\
\text { without IgG }\end{array}$ & $\begin{array}{l}\text { Culture } \\
\text { condition: } \\
\text { with IgG } \\
\end{array}$ & $\begin{array}{l}\text { Culture } \\
\text { condition: } \\
\text { without IgG }\end{array}$ & $\begin{array}{l}\text { Culture } \\
\text { condition: } \\
\text { without IgG }\end{array}$ & $\begin{array}{l}\text { Culture } \\
\text { condition: } \\
\text { without IgG }\end{array}$ & $\begin{array}{l}\text { Culture } \\
\text { condition: } \\
\text { without IgG }\end{array}$ \\
\hline 2000 & $160 \pm 0$ & $<10 \pm 0$ & $10 \pm 0$ & $<4 \pm 0$ & $<4 \pm 0$ & $<4 \pm 0$ & $<4 \pm 0$ \\
\hline 2003 & $160 \pm 0$ & $<10 \pm 0$ & $10 \pm 0$ & $<4 \pm 0$ & $<4^{a}$ & $<4 \pm 0$ & $<4 \pm 0$ \\
\hline 2006 & $160 \pm 0$ & $<10 \pm 0$ & $10 \pm 0$ & $<4 \pm 0$ & $<4^{a}$ & $<4 \pm 0$ & $<4 \pm 0$ \\
\hline 2007 & $160 \pm 0$ & $<10 \pm 0$ & $10 \pm 0$ & $<4 \pm 0$ & $<4 \pm 0$ & $<4 \pm 0$ & $<4 \pm 0$ \\
\hline 2008 & $160 \pm 0$ & $<10 \pm 0$ & $10 \pm 0$ & $<4 \pm 0$ & $<4 \pm 0$ & $<4 \pm 0$ & $<4 \pm 0$ \\
\hline 2009 & $160 \pm 0$ & $<10 \pm 0$ & $10 \pm 0$ & $<4 \pm 0$ & $<4 \pm 0$ & $<4 \pm 0$ & $<4 \pm 0$ \\
\hline 2010 & $160 \pm 0$ & $<10 \pm 0$ & $10 \pm 0$ & $<4 \pm 0$ & $<4 \pm 0$ & $<4 \pm 0$ & $<4 \pm 0$ \\
\hline
\end{tabular}

Notes: Results are shown as mean \pm SE by year of several products $(n=3) .{ }^{a} n=I$. Cultures were done either with lgG or without lgG. Serotypes: type I, clinical isolate; type 2, New Guinea C strain; type 3, clinical isolate; and type 4, clinical isolate.

Abbreviations: DenV, dengue virus; IG, immunoglobulin G; IVIG, intravenous immunoglobulin; JEV, Japanese encephalitis virus; SE, standard error; WNV, West Nile virus.

animal study indicated that JEV vaccination could potentially induce protection against West Nile viremia or encephalitis. ${ }^{10}$ However, no human cross-reactive antibody against WNV was observed in samples of donors who had received the JEV vaccination. ${ }^{11}$ Similarly, the results of the present study showed no or very weak activity against WNV and DenV in the IVIG samples. This suggests that the epidemiological level of WNV and DenV of the donor population is still low in Japan, implying that these viruses are not yet indigenized. In addition, JEV vaccinations and/or infections in the donor population did not induce a cross-reactive antibody against WNV. In general, IVIG products are manufactured only after at least 1 year following the blood donation. Therefore, these results suggest that WNV and DenV had not yet indigenized the donor population in Japan from 2000 to around 2008.

\section{Acknowledgments}

This study was partially conducted as a collaborative research project between Osaka University, Rakuno Gakuen University, Osaka Prefectural Institute of Public Health, The Research Foundation for Microbial Diseases of Osaka University, and The Japan Blood Products Organization (formerly Benesis Corporation).

\section{Disclosure}

M Yunoki is an employee of Japan Blood Products Organization (formerly Benesis Corporation). RK Koketsu and Y Okuno were employees of The Research Foundation for Microbial Diseases of Osaka University during the study period. The authors report no conflicts of interest in this work.

\section{References}

1. King AMQ, Adams MJ, Carstens EB, Lefkowitz EJ. Ninth Report of the International Committee on Taxonomy of Viruses. London: Elsevier Academic Press; 2012.

2. Campbell GL, Hills SL, Fischer M, et al. Estimated global incidence of Japanese encephalitis: a systematic review. Bull World Health Organ. 2011;89:766-774.

3. Fischer M, Lindsey N, Staples JE, Hills S; Center for Disease Control and Prevention (CDC). Japanese encephalitis vaccines: recommendations of the Advisory Committee on Immunization Practices (ACIP). MMWR Recomm Rep. 2010;59(RR-1):1-27.

4. Infectious disease surveillance in Japan 2013 [in Japanese]. Available from: http://www.nih.go.jp/niid/ja/survei/2085-idwr/ydata/5194-reportja2013-20.html. Accessed May 31, 2016.

5. Arima Y, Matsui T, Shimada T, et al. Ongoing local transmission of dengue in Japan, August to September 2014. Western Pac Surveill Response J. 2014;5(4):27-29.

6. Planitzer CB, Modrof J, Yu MW, Kreil TR. West Nile virus infection in plasma of blood and plasma donors, United States. Emerg Infect Dis. 2009; 15(10):1668-1670.

7. Jirakanjanakit N, Sanohsomneing T, Yoksan S, Bhamarapravati N. The micro-focus reduction neutralization test for determining dengue and Japanese encephalitis neutralizing antibodies in volunteers vaccinated against dengue. Trans R Soc Trop Med Hyg. 1997;91(5): 614-617.

8. Lim CK, Takasaki T, Kotaki A, Kurane I. Vero cell-derived inactivated West Nile (WN) vaccine induces protective immunity against lethal WN virus infection in mice and shows a facilitated neutralizing antibody response in mice previously immunized with Japanese encephalitis vaccine. Virology. 2008;374:60-70.

9. Masrinoul P, Omokoko MD, Pambudi S, Ikuta K, Kurosu T. Serotypespecific anti-dengue virus NS1 mouse antibodies cross-react with $\mathrm{prM}$ and are potentially involved in virus production. Viral Immunol. 2013;26(4):250-258.

10. Takasaki T, Yabe S, Nerome R, Ito M, Yamada K, Kurane I. Partial protective effect of inactivated Japanese encephalitis vaccine on lethal West Nile virus infection in mice. Vaccine. 2003;21:4514-4518.

11. Kanesa-Thasan N, Putnak JR, Mangiafico JA, Saluzzo JE, Ludwig GV. Short report: Absence of protective neutralizing antibodies to West Nile Virus in subjects following vaccination with Japanese encephalitis or dengue vaccines. Am J Trop Med Hyg. 2002;66(2):115-116. 


\section{Publish your work in this journal}

Biologics: Targets and Therapy is an international, peer-reviewed journal focusing on the patho-physiological rationale for and clinical application of Biologic agents in the management of autoimmune diseases, cancers or other pathologies where a molecular target can be identified. This journal is indexed on PubMed Central, EMBase, and Scopus.
The manuscript management system is completely online and includes a very quick and fair peer-review system, which is all easy to use Visit http://www.dovepress.com/testimonials.php to read real quotes from published authors. 